\title{
A repulsive trap for two electrons in a magnetic field
}

\author{
A. D. Chepelianskii ${ }^{(a)}$ and D. L. Shepelyansky ${ }^{(b)}$ \\ (a) Lycée Pierre de Fermat, Parvis des Jacobins, 31068 Toulouse Cedex 7, France \\ (b) Laboratoire de Physique Quantique, UMR 5626 du CNRS, Université Paul Sabatier, 31062 Toulouse Cedex 4, France
}

(November 1, 2000)

\begin{abstract}
We study numerically and analytically the dynamics of two classical electrons with Coulomb interaction in a two dimensional antidot superlattice potential in the presence of crossed electric and magnetic fields. It is found that near one antidot the electron pair can be trapped for a long time and the escape rate from such a trap is proportional to the square of a weak electric field. This is qualitatively different from the case of noninteracting electrons which are trapped forever by the antidot. For the pair propagation in the antidot superlattice we found a broad parameter regime for which the pair is stable and where two repulsive electrons propagate together on an enormously large distance.
\end{abstract}

PACS numbers: 03.20.+i, 05.45.Mt, 72.20.My

\section{INTRODUCTION}

Recent technological development allowed to create various types of surface superlattices for two-dimensional (2D) electron gas in semiconductor heterostructures and to investigate their transport properties in presence of magnetic field. Experiments with antidot lattices yere carried out by different experimental groups (see e.g. and the contribution of classical periodic orbits in the resistivity peaks at certain values of magnetic field was clearly identified. In fact the size of antidots and the distance between them are relatively large, and analysis of classical trajectory dynamics can be successfully applied to understand a number ff unusual transport properties in such antidot arrays 6 . Due to nonlinearity of motion in the vicinity of antidot potential the classical dynamics can be chaotic that leads to diffusive spread ing of trajectories even for perfectly periodic lattices 5 . If the distance between antidots is large or comparable to the cyclotron radius of an electron in a magnetic field perpendicular to the lattice, then one should understand first, the properties of electron dynamics near one antidot. In the absence of electric field, the dynamics is integrable for an antidot of circular shape due to angular momentum conservation and an electron always regularly rotates around the antidot. An electric field applied in the 2D plane of the superlattice breaks the cylindrical symmetry and can lead to the electron escape from the antidot to infinity. The problem of electron dynamics in crossed electric $E$ and magnetic $B$ fields near a circular elastic disk (antidot) was studied int. It was shown that the dynamics can be described by a simple area-preserving map which depends only on one dimensionless parameter $\epsilon=(2 \pi m / a e)\left(E / B^{2}\right)$, where $m, e$ are electron mass and charge, and $a$ is the disk radius. For small $\epsilon<\epsilon_{c}$ the electron dynamics in the phase space of angular momentum $l$ and conjugated angle $\phi$ is bounded by the invariant Kolmogorv-Arnold-Moser (KAM) curves so that electron always remains near the disk. On the contrary for $\epsilon>\epsilon_{c}$ the KAM curves are destroyed, global chaos sets in and the electron escapes to infinity after few collisions with the disk.

Until now the classical dynamics in antidgt lattices was studied only for noninteracting electrons $\mathrm{O}$ - In this paper for the first time we analyze the effect of Coulomb interaction between classical electrons in the vicinity of an antidot. We show that for sufficiently strong interaction between electrons their dynamics becomes chaotic. Due to that one or two electrons can escape from the antidot even in an arbitrary weak applied electric field $E$ that corresponds to $\epsilon_{c} \rightarrow 0$ contrary to $\epsilon_{c}>0$ in absence of interaction. We determine the dependence of average escape rate $\Gamma$ on $\epsilon$ showing that in the limit of small electric field $\Gamma \propto \epsilon^{2}$. After that we also discuss the two electron propagation in the antidot superlattice.

The paper is organized as follows. In the next Section we briefly discuss the one electron dynamics near the antidot in crossed magnetic and electric fields. In Section III the dynamics of two interacting electrons is analyzed in detail. The electron motion in the antidot superlattice is considered in Section IV. In the last Section we summarize the obtained results.

\section{ONE ELECTRON DYNAMICS}

The dynamics of an electron in crossed electric and magnetic fields in two dimensions with one antidot is described by the Hamiltonian:

$$
H_{0}=(\mathbf{p}-e \mathbf{A})^{2} / 2 m+U(x, y)-e \mathbf{E r}
$$

where $\mathbf{A}$ is the vector potential and $U(x, y)$ describes the antidot potential which depends only on the radius $r=\sqrt{x^{2}+y^{2}}$ with $U=0$ for $r \geq a$. For convenience, following 0 , we introduce the dimensionless variables $\tilde{x}=x / a, \tilde{y}=y / a, \tilde{t}=t / \tau_{0}, \tilde{H}_{0}=H_{0} / 2 \epsilon_{F}, \tilde{U}=$ $U / 2 \epsilon_{F}, \tilde{B}=B / B_{0}, \tilde{E}=E / E_{0}$ where $\epsilon_{F}\left(v_{F}\right)$ is the Fermi energy (velocity), $\tau_{0}=\left(2 \epsilon_{F} / m a^{2}\right)^{-1 / 2}=a / v_{F}$, magnetic and electric fields are scaled by $B_{0}=\left(m \epsilon_{F}\right)^{1 / 2} / e a$ and 
$E_{0}=2 \epsilon_{F} /$ ea respectively. In these units a magnetic field $B=B_{0}$ gives the cyclotron radius $R_{c}=a$ for electron with energy $\epsilon_{F}=m v_{F}^{2} / 2$. We choose the Landau gauge $\mathbf{A}=(-B y, 0,0)$. Then omitting the tildes the Hamiltonian equations of motions reads:

$$
\begin{aligned}
& d x / d t=v_{x}, \quad d v_{x} / d t=B v_{y}-d U / d x-E_{x} \\
& d y / d t=v_{y}, \quad d v_{y} / d t=-B v_{x}-d U / d y-E_{y}
\end{aligned}
$$

where $v_{x}=p_{x}+y B, v_{y}=p_{y}$. To model the antidot we chose the potential:

$$
U(x, y)=U_{0}(1-r)^{6} .
$$

Usually we choose $U_{0}$ to be much larger than the electron energy $H_{0}$ so that this potential becomes very similar to an absolutely rigid disk with effective radius $a_{e f f}$ about $15 \%$ smaller than $a$.

Far from the antidot the equations of motion are exactly solvable and give electron rotation over a circle of cyclotron radius $R_{c}=v / \omega_{c}$ with cyclotron frequency $\omega_{c}=B$. In addition this circle moves with the drift velocity $v_{d}=E / B$ in the direction perpendicular to electrif and magnetic fields. As it was found by Berglund et al. . near the antidot, the dynamics strongly depends on the dimensionless parameter $\epsilon=v_{d} 2 \pi / \omega_{c}=2 \pi E / B^{2}$. For $\epsilon \ll 1$ the electron scatters on the antidot and escapes to the infinity after one collision. On the confrary, the situation with not very large $\epsilon$ is much richert. In this case electron can collide many times with the antidot and this process is described by a simple area-preserving mapt:

$$
\begin{gathered}
\bar{\phi}=\phi+\pi-2 \sin ^{-1} \beta \\
\bar{\beta}=\beta-\epsilon \sin \bar{\phi}
\end{gathered}
$$

where bars denote the new values of variables after collision, $\phi$ is the scattering angle measured in respect to the direction of drift velocity and $\beta$ is the scattering impact parameter divided by the antidot radius. In this way $\beta$ varies in the interval $(-1,1)$. We note that $\beta$ can be also considered as the orbital momentum $l$ of the electron divided by the maximal momentum $l_{\max }=a v$ at which electron still collides with the antidot. The real dynamics is correctly described by the map if $R_{c} \gg 1$, that corresponds to $v \gg B$. For $\epsilon \gg 1$ the variation of $\beta$ is bounded by the invariant KAM curves and electron is trapped near the antidgt. The last KAM curve is destroyed for $\epsilon>\epsilon_{c} \approx 0.45$ so that the orbits with initial $\beta \approx 0$ can escape from the antidot to infinity. Of course, for $\epsilon>\epsilon_{c}$ some islands with regular motion inside still remain, but they become very small as soon as $\epsilon$ becomes significantly lager than $\epsilon_{c}$.

To study the electron dynamics in (1) the Hamiltonian equations of motion are solved numerically by RungeKutta method of fourth order so that the electron energy is conserved with the relative precision better than $10^{-6}$. The examples of the Poincaré cross sections constructed at $x=0$ and $v_{x}>0$ for trajectories trapped near the antidot is shown in Figs. 1,2. In Fig. $1 \epsilon \approx 0.16$ is rather small and almost all phase space is filled by integrable KAM curves. For Fig. 2, the parameter $\epsilon \approx 0.42$ is close to $\epsilon_{c}$ and KAM curves become more deformed and the chaotic component becomes visible. This case can be compared with Fig. 3 in where the cross section for the map (4) is given for a close value of $\epsilon$.

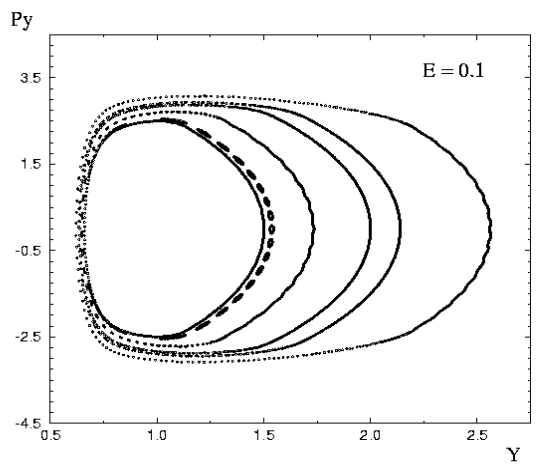

FIG. 1. Poincaré cross section for the Hamiltonian (1) constructed at $x=0, v_{x}>0$ for $H_{0}=8.725, B=-2, E=0.1$ so that $\epsilon \approx 0.16$. The antidot determined by the potential (3) is located at $(0,0) ; U_{0}=1000$.

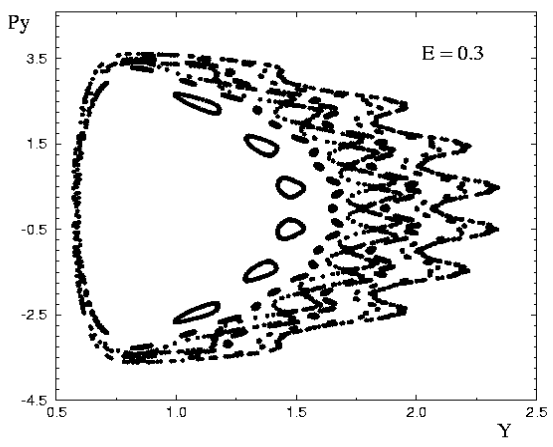

FIG. 2. Same as in Fig. 1 but for $E=0.3, \epsilon \approx 0.42$.

It is interesting to note that the map (何 can be not valid if orbits have $|\beta| \approx 1$ or $|\beta|>1$. For example, if the antidot is inside a large cyclotron circle then electron will make many many rotations before this slowly drifting circle will cross the antidot. This situation is not taken into account by the first equation in (4). An example of the electron dynamics in this case is given in Fig. 3. Here $\epsilon \approx 0.16$ is small and the motion is still regular. We should stress that such type of trajectories separates orbits which escape to infinity and those which collide with the antidot on each cyclotron period. For the antidot superlattice with antidot spacing comparable with 
$R_{c}$ this type of orbits (see Fig. 3) is of special importance since these orbits can easily jump from one antidot to another leading to a global diffusion in the system. We will discuss this situation in Section III.

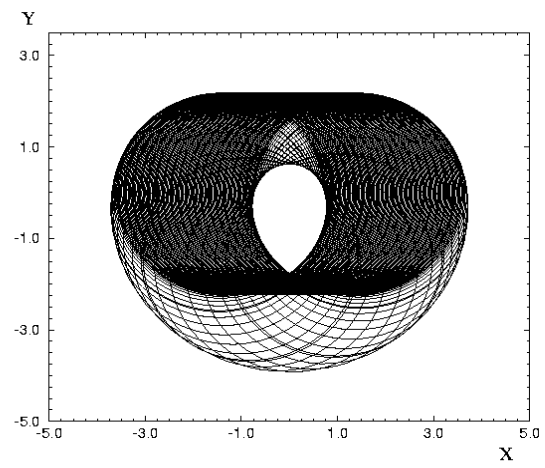

FIG. 3. Electron dynamics in $(x, y)$ plane near the antidot of Fig. 1 for $H_{0}=9.7, B=-2.0, E=0.1, \epsilon \approx 0.16$.

\section{EFFECTS OF COULOMB INTERACTION ON ELECTRON DYNAMICS}

Let us now consider how the Coulomb interaction between two electrons affects their dynamics near the antidot. In this case the Hamiltonian of the system reads

$$
H=H_{0}\left(\mathbf{p}_{\mathbf{1}}, \mathbf{r}_{\mathbf{1}}\right)+H_{0}\left(\mathbf{p}_{\mathbf{2}}, \mathbf{r}_{\mathbf{2}}\right)+e^{2} /\left|\mathbf{r}_{\mathbf{1}}-\mathbf{r}_{\mathbf{2}}\right| .
$$

While in the free space the Coulomb interaction repels the electrons and leads to their separation the situation is more complicated in the presence of magnetic field. In the case without any antidot the total momentum of two electrons is conserved and as a result each electron rotates regularly on a cyclotron circle which in addition rotates around the center of mass of the system. Without an external electric field $(E=0)$ the center of mass is fixed and inert whereas in the presence of the field $(|E|>0)$ the center of mass drifts with the constant velocity $v_{d}=E / B$ but the average distance between electrons remains constant. However this electron pair can be trapped by the repulsive potential of the antidot so that the electrons will spend a long time colliding with this antidot. An example of electron dynamics in this case is shown in Fig. 4. It shows that an electron can escape from the antidot even in the situation with $\epsilon<\epsilon_{c}$ when without the interaction the electrons remain trapped near the antidot. In our numerical simulations we observed different cases where one or both of the electrons escape to infinity.
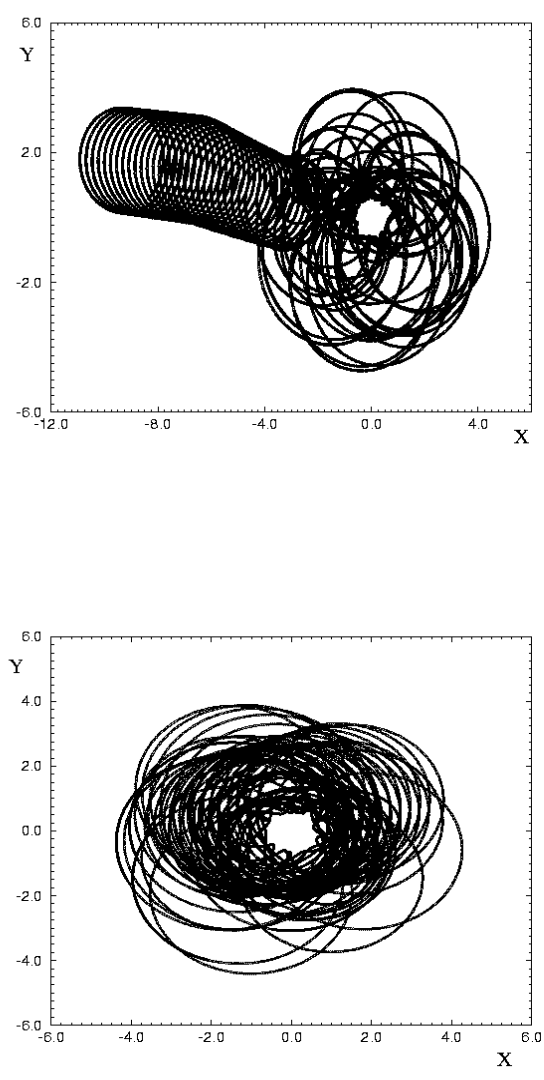

FIG. 4. Dynamics of two electrons in $(x, y)$ plane for $H=15.65, B=-2.0, E=0.15, \epsilon \approx 0.24$ and initial distance between electrons $\left|\mathbf{r}_{1}-\mathbf{r}_{\mathbf{2}}\right| \approx 0.5$. After many cyclotron periods the first electron escapes from the antidot to infinity (upper figure) while the second remains trapped forever (bottom figure).

To investigate how the escape rate depends on the strength of an external electric field $E$ we studied the ensemble of 100 paths. In each path the positions and momentums of each electron are chosen randomly in the intervals $-4 \leq x, y \leq 4,-2 \leq p_{x}, p_{y} \leq 2$ in such a way that the total energy is $H \approx 15 \pm 0.5$. We remind that the antidot with potential (3) is placed at $(0,0)$ and $U_{0}=1000$. The escape rate $\Gamma$ is defined as $\Gamma=1 / T$ where $T$ is the time after which the distance of one of the electrons from the antidot is greater than $R_{e s c} \approx 5 R_{c} \approx 10$. This distance is sufficiently large and as soon as it is reached an electron escapes to infinity and never returns back to the antidot. The average value of $\Gamma$ is obtained by averaging over 100 values obtained for 100 randomly chosen paths.

The dependence of the escape rate $\Gamma$ on the strength of the applied electric field $E$ is presented in Fig. 5. It definitely shows that the escape takes place even at very weak electric fields with $\epsilon \ll \epsilon_{c}$ when without Coulomb interaction electrons are forever trapped near the antidot. According to the obtained numerical data (see Fig. 5) in 
the limit of $\epsilon \rightarrow 0$ the escape rate is

$$
\Gamma / \omega_{c} \approx \epsilon^{2} .
$$

Our understanding of this dependence is based on the following argument. Due to the Coulomb interaction between electrons their dynamics in the vicinity of the antidot becomes chaotic. Therefore the phase $\phi$ in the map (画) changes randomly between electron collisions with the antidot and $\beta$ grows diffusively with the number of collisions $n$ so that $(\Delta \beta)^{2} \approx D n$ with $D=\epsilon^{2} / 2$. This diffusion results in the escape rate $\Gamma / \omega_{c} \sim D \sim \epsilon^{2}$ being in agreement with the numerical data in Fig. 5.

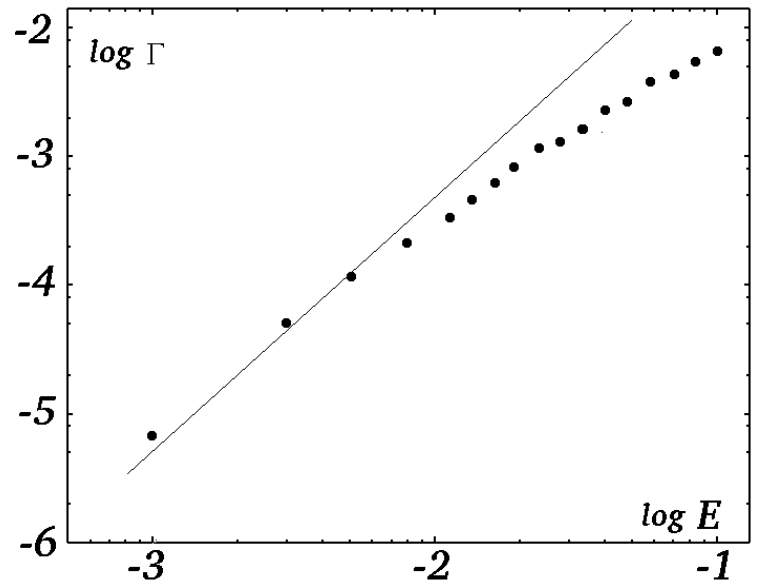

FIG. 5. Dependence of the escape rate $\Gamma$ on the electric field $E$ for electrons being initially in the vicinity of the antidot at $B=-2.0, H \approx 15.0 \pm 0.5, U_{0}=1000$. Here averaging is done over 100 paths, $\omega_{c}=2, \epsilon=2 \pi E / B^{2}$, points give the numerical results for $\Gamma$ and the straight line gives the dependence (6). Logarithms are decimal.

\section{TWO ELECTRON PROPAGATION IN ANTIDOT SUPERLATTICE}

We also studied the electron dynamics on a square antidot superlattice, when the antidot potential is given by (3) and the distance between antidots $d>2$. In this case our results for one electron dynamics are in qualitative agreement with the conclusions drawn in 5 . As soon as the cyclotron radius $R_{c}$ becomes comparable with the antidot spacing $d$ the trajectories start to move diffusively on the whole lattice. Trapped orbits near one antidot exist only for $2 R_{c}<d$ and $\epsilon<\epsilon_{c}$. For $R_{c}>d / 2$ the cyclotron circle starts to drift in a way similar to that one shown in Fig. 3. After a time $t_{d} \sim d / v_{d}$ a collision with another antidot takes place that finally originates a sequence of irregular jumps between antidots. The diffusion rate on the superlattice originated by this process can be estimated as $D_{\text {lat }} \sim d^{2} / t_{d} \sim d v_{d}$. This diffusion is important in the limit $R_{c} \sim d \gg 1$. However, we note that even at $E=0$ at $R_{c}>d / 2$ there are chaotic orbits which diffyse over the whole lattice as it was discussed in detail in 5 - 6 and this diffusion is dominant for $d \sim 1$.

It is interesting to understand how two electrons move in such a superlattice. Intuitively, one would expect that the Coulomb repulsion will separate electrons and they will not propagate together. In fact we found that it is not necessarily the case and there are regimes where two electrons propagate together. An example of such a case is shown in Fig. 6. In this case the electron pair moves with an average drift velocity $v_{d} \approx E / B$ and the total displacement of the pair is about hundred times larger than the distance between the two electrons (Fig. 6).
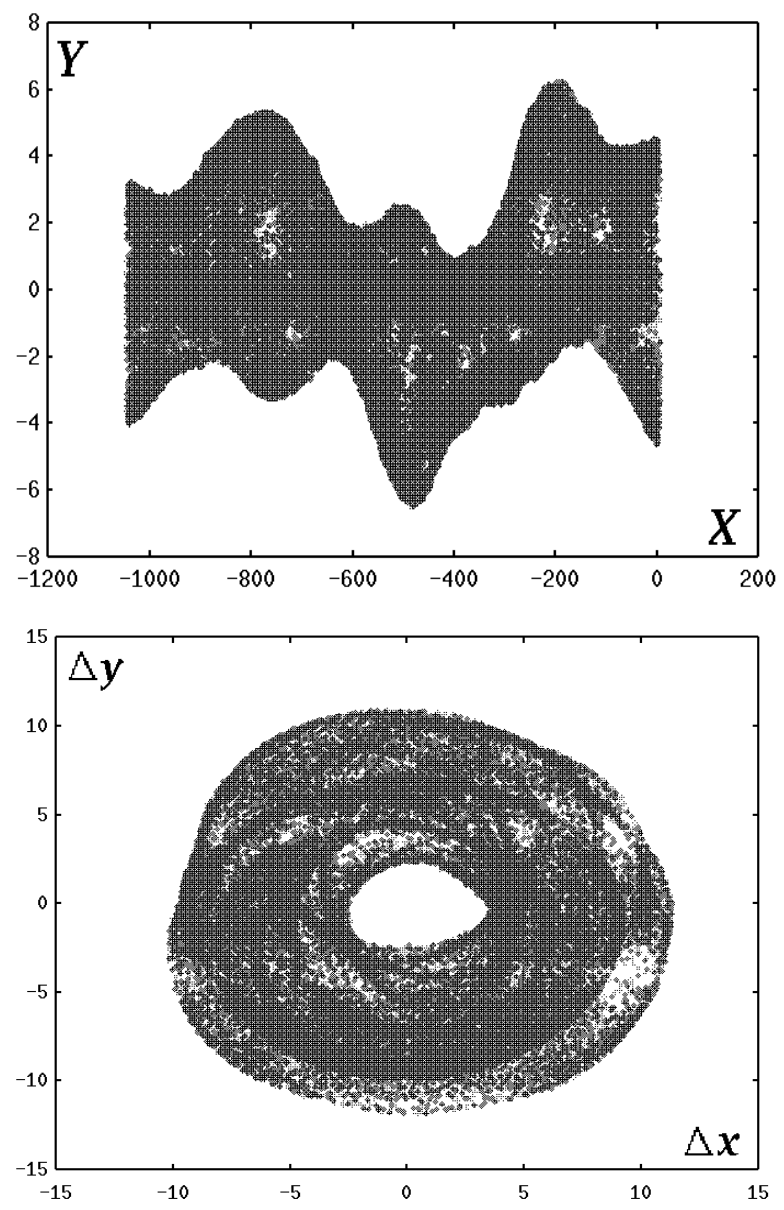

FIG. 6. Dynamics of two electrons in the $(x, y)$ plane of an antidot superlattice for $H \approx 51, B=-2.0, E=0.4, \epsilon \approx 0.63$ and initial distance between electrons $\left|\mathbf{r}_{1}-\mathbf{r}_{\mathbf{2}}\right| \approx 4$. The antidots are placed on a square superlattice with period 4 and $U_{0}=1$ in (3). The upper figure shows the propagation of the first electron in the plane $(x, y)$, while the bottom figure shows the distance between the electrons $\Delta x=x_{1}-x_{2}, \Delta y=y_{1}-y_{2}$.

The physical reason for appearance of such electron pairs is quite clear in the absence of superlattice potential. In this case, as it was discussed before at the beginning of Section III, electrons rotate one around another and propagate together, their dynamics is integrable. Then according to the KAM theorem a weak 
perturbation will not destroy such pairs. Indeed, in the case of Fig. 6 the antidot potential is relatively weak $\left(U_{0}=1 \ll H / 2 \approx 25\right)$ and the pair is not destroyed. We checked numerically that provided $U_{0} \sim H / 2$ the pair size starts to grow diffusively due to random scattering on a strong antidot potential, and eventually the pair is destroyed and electrons continue to propagate separately. For $U_{0} \gg H$ the separation happens after a few collisions with antidots. On the contrary for $U_{0} \ll H$ the life time of the classical pair becomes infinite in agreement with the KAM theorem.

\section{CONCLUSIONS}

In this paper we investigated the effects of Coulomb interaction between two electrons on their classical dynamics in antidot superlattice in crossed electric and magnetic fields. We found that for weak electric field the electron pair can be trapped for a long time near an antidot even if eventually one or two electrons escape from the antidot. The escape rate $\Gamma$ decreases proportionally to the square of electric field. This behaviour is qualitatively different from the case of noninteracting electrons which are trapped forever near the antidot in the limit of small electric field.

The study of the electron pair dynamics in the antidot superlattice showed that the Coulomb repulsion can create stable pairs propagating on a large distance. In agreement with the KAM theorem such pairs are stable when the antidot potential strength is relatively weak compared to the electron energy. On the contrary, in the opposite limit the pairs become unstable and electrons are quickly separated from one another. On the basis of this phenomenon it is possible to make a conjecture that in two dimensional heterostructures with high mobility the impurity potential is relatively weak and such electron KAM pairs will be stable and can be detected experimentally. We note that in the recent experiments 8 with $2 \mathrm{D}$ electron gas the carriers of charge $2 e$ have been detected. It is possible that these carriers are related to the KAM pairs found in this paper.

We thank G. Gasati who attracted our interest to the results found ind.

${ }^{1}$ K. Ensslin and P. M. Petroff, Phys. Rev. B 41, 12307 (1990).

${ }^{2}$ D. Weiss, M. L. Roukes, A. Mesching, P. Grambow, K. von Klitzing, G. Weiman, Phys. Rev. Lett. 66, 2790 (1991).

${ }^{3}$ G. M. Gusev, V. T. Dolgopolov, Z. D. Kvon, A. A. Shashkin, V. M. Kudryashov, L. V. Litvin and Yu. Nastaushev, Pis'ma Zh. Eksp. Teor. Fiz. 54, 369 (1991).

${ }^{4}$ M. V. Budantsev, Z. D. Kvon, A. G. Pogosov, G. M. Gusev, J. C. Portal, D. K. Maude, N. T. Moshegov and A. I. Toropov, Physica B 256-258, 595 (1998).
${ }^{5}$ R. Fleischmann, T. Geisel, and R. Ketzmerick, Phys. Rev. Lett. 68, 1367 (1992).

${ }^{6}$ T. Geisel, R. Ketzmerick and O. Schedletzky, Phys. Rev. Lett. 69, 1680 (1992).

${ }^{7}$ N. Berglund, A. Hansen, E. H. Hauge, and J. Piasecki, Phys. Rev. Lett. 77, 2149 (1996).

${ }^{8}$ D. C. Glattli, V. Rodriguez, H. Perrin, P. Roche, Y. Jin and B. Etienne, Physica E 6, 22 (2000). 